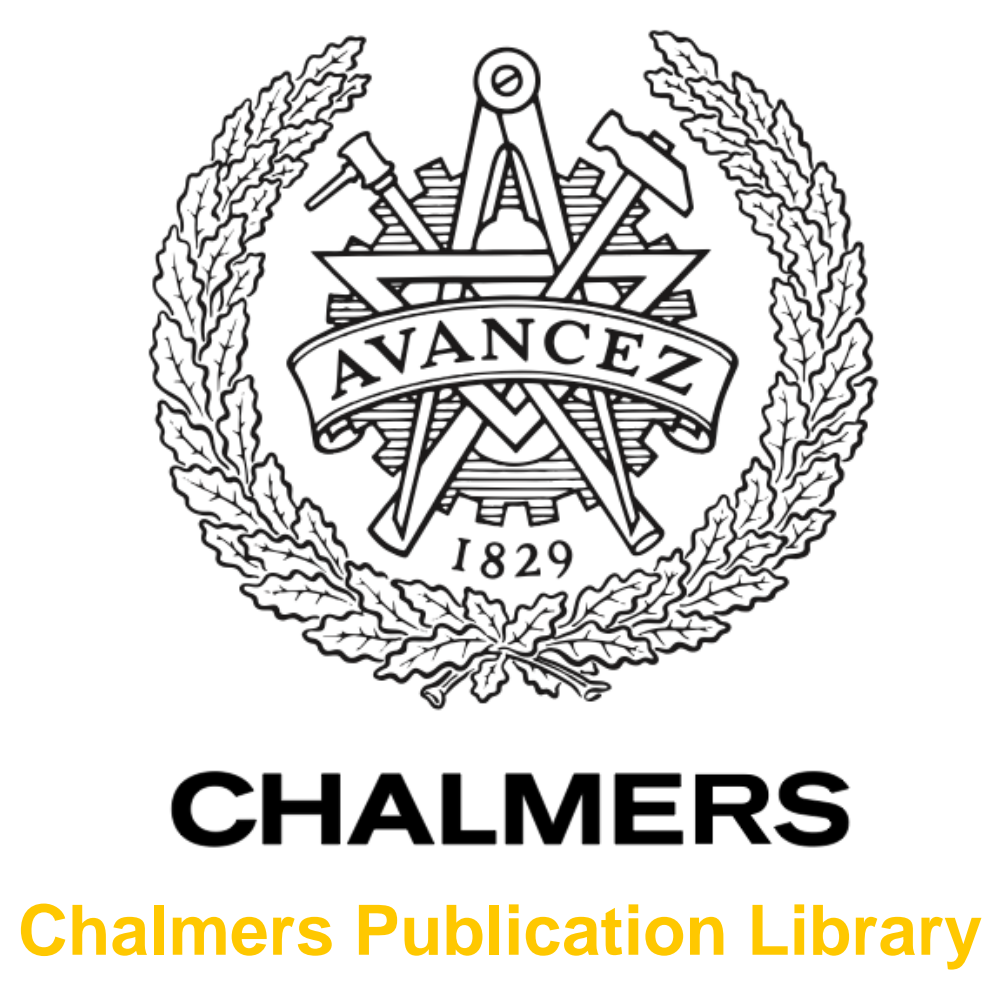

\title{
Dynamic equations for a fully anisotropic elastic plate
}

This document has been downloaded from Chalmers Publication Library (CPL). It is the author's version of a work that was accepted for publication in:

Journal of Sound and Vibration (ISSN: 0022-460X)

Citation for the published paper:

Mauritsson, K. ; Folkow, P. ; Boström, A. (2011) "Dynamic equations for a fully anisotropic elastic plate". Journal of Sound and Vibration, vol. 330(11), pp. 2640-2654.

http://dx.doi.org/10.1016/j.jsv.2010.12.016

Downloaded from: http://publications.lib.chalmers.se/publication/139930

Notice: Changes introduced as a result of publishing processes such as copy-editing and formatting may not be reflected in this document. For a definitive version of this work, please refer to the published source. Please note that access to the published version might require a subscription.

Chalmers Publication Library (CPL) offers the possibility of retrieving research publications produced at Chalmers University of Technology. It covers all types of publications: articles, dissertations, licentiate theses, masters theses, conference papers, reports etc. Since 2006 it is the official tool for Chalmers official publication statistics. To ensure that Chalmers research results are disseminated as widely as possible, an Open Access Policy has been adopted.

The CPL service is administrated and maintained by Chalmers Library. 


\title{
DYNAMIC EQUATIONS FOR A FULLY ANISOTROPIC ELASTIC PLATE
}

\author{
KARL MAURITSSON, PETER D. FOLKOW, ANDERS BOSTRÖM
}

\begin{abstract}
A hierarchy of dynamic plate equations is derived for a fully anisotropic elastic plate. Using power series expansions in the thickness coordinate for the displacement components, recursion relations are obtained among the expansion functions. Adopting these in the boundary conditions on the plate surfaces and along the edges, a set of dynamic equations with pertinent edge boundary conditions are derived on implicit form. These can be truncated to any order and are believed to be asymptotically correct. As a special case, explicit equations for an orthotropic plate are presented. Analytical and numerical comparisons are made with other approximate theories given in the literature. These results show that the present theory capture the plate behavior accurately concerning dispersion curves, eigenfrequencies as well as stress and displacement distributions.
\end{abstract}

\section{INTRODUCTION}

Vibrations of anisotropic plates have been studied extensively by many authors. As the 3D equations of motion result in solutions that generally are of a considerably higher complexity than in the isotropic case [1,2], the main interest has concerned approximate plate theories. Here the bulk of work deals with the special case of orthotropic plates. This is partly due to the fact that the involved constitutive relations in a general anisotropic material results in quite complicated plate equations even for an approximate theory. Moreover, in many engineering applications using composites the material configuration is orthotropic.

Among the approximate plate equations, the most used theory is the classic bending equation based on the Kirchhoff assumptions [3]. It is well known that this theory models the lowest flexural mode that leads to good results in the long wave region. The more accurate theory due to Mindlin [4] includes effects of rotatory inertia and shear. Other more refined theories has been developed ever since $[5,6,7,8,9,10,11]$. Recently, a Timoshenko like plate theory has been developed by Shimpi and Patel [12].

Power series expansion techniques truncated to arbitrary order have been used by several authors, both for isotropic plates $[13,14,15,16,17,18,19,20]$ and anisotropic plates $[21,22]$. Among these, the important concept of developing recursion relations appears in $[13,15,17,19,20]$, from which it is possible to reduce the number of displacement fields in a consistent manner. The lateral boundary conditions are in addition exactly fulfilled in these latter works since the surface boundary conditions actually constitute the plate equations of motion. However, there are also several differences in the derivation procedure among the papers using recursion relations, such as in the series expansion formulation or in the truncation process. Considering the cited works on anisotropic plates, Mindlin [21] and Yu [22] have developed sets of higher order equations without adopting recursion relations. In 
the truncation process described in [21], certain displacement terms are simply omitted to reduce the number of unknowns. Moreover, the lateral boundary conditions are not fulfilled exactly. The present paper is based on the method used for isotropic plates by Boström et al. [20]. This particular method has previously been adopted to other plate structures such as piezoelectric plates [23, 24] and porous plates [25] (the latter work is influenced by Losin's method [19]). Moreover, several papers on different structural elements have also been addressed in the same fashion: isotropic rods [26, 27], anisotropic rods [28, 29] and shells [30].

Here, dynamic plate equations for a homogenous, fully anisotropic, elastic plate are derived by adopting a systematic power series expansion approach. Assuming power series expansions in the thickness coordinate for the displacement components, the governing three-dimensional equations of motion results in recursion relations among the expansion functions. These can be used to eliminate all but the six lowest order expansion functions. Next, the power series expansions in conjunction with the recursion relations are used in the surface boundary conditions at the top and the bottom of the plate; either expressed in terms of prescribed displacements or prescribed stresses. The resulting set of six scalar equations constitutes the dynamic plate equations of motion. By adopting variational calculus, the pertinent edge boundary conditions are stated in terms of the six lowest order fields. These final plate equations can be truncated to arbitrary order in the thickness, resulting in a hierarchy of plate theories that are believed to be asymptotically correct.

One advantage with the consideration of a material without any symmetry classes is that all other cases can be obtained as special cases. However, as the stiffness matrix for a fully anisotropic material includes 21 independent stiffness constants resulting in very complicated explicit expressions for the plate equations, these equations are here expressed on implicit form in terms of recursively defined differential operators. Due to these involved expressions, it is natural to study a simpler material configuration more in detail, so as to obtain explicit sets of plate equations. Hence, the special case of an orthotropic material with standard surface boundary conditions is considered. Here the six plate equations can be added and subtracted in pairs to obtain two uncoupled systems of equations, each of them including three equations and three unknowns. The two uncoupled systems correspond to the symmetric and antisymmetric part of the motion, respectively. These equations, including the edge boundary conditions, are explicitly given to cubic order in the thickness, which thus accounts for the bending stiffness. For the antisymmetric case, analytical comparisons are performed with approximate theories appearing in the literature. To validate the antisymmetric plate equations, dispersion curves, eigenfrequencies as well as displacement and stress distribution curves are presented. These are compared to other approximate theories as well as the exact three-dimensional theory.

\section{GOVERNING EQUATIONS}

Consider a plate of thickness $2 h$ according to Fig. 1. The plate is homogenous, fully anisotropic and linearly elastic with density $\rho$. Introducing the notation used by Auld [31], the governing equations of motion are written

$$
\nabla_{i J} T_{J}=\rho \partial_{t}^{2} u_{i}
$$




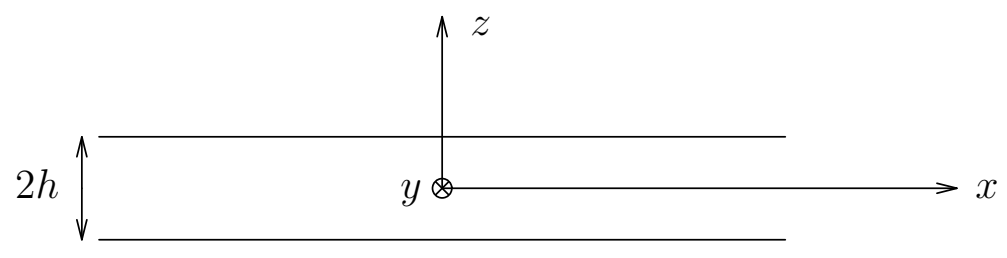

Figure 1. The geometry.

Here, the stress and displacement column matrices are defined through

$$
\left[T_{I}\right]=\left(\begin{array}{llllll}
T_{x x} & T_{y y} & T_{z z} & T_{y z} & T_{x z} & T_{x y}
\end{array}\right)^{T}, \quad\left[u_{i}\right]=\left(\begin{array}{lll}
u & v & w
\end{array}\right)^{T},
$$

respectively, where the displacement components are in the $x, y$ and $z$ directions. Note that vector subscripts are expressed through lower case letters $i=x, y, z$, while abbreviated subscripts are expressed through upper case letters $I=1,2,3,4,5,6$. The stresses are related to the displacements through

$$
T_{I}=c_{I J} \nabla_{J k} u_{k}
$$

where the symmetric stiffness matrix, denoted by $c_{I J}$, includes the 21 independent stiffness constants. The divergence of stress operator $\nabla_{i J}$ and the symmetric gradient operator $\nabla_{I j}$ are defined by the matrix representations

$$
\left[\nabla_{i J}\right]=\left(\begin{array}{cccccc}
\partial_{x} & 0 & 0 & 0 & \partial_{z} & \partial_{y} \\
0 & \partial_{y} & 0 & \partial_{z} & 0 & \partial_{x} \\
0 & 0 & \partial_{z} & \partial_{y} & \partial_{x} & 0
\end{array}\right), \quad\left[\nabla_{I j}\right]=\left[\nabla_{i J}\right]^{T}
$$

Here, the partial derivatives are expressed as $\partial_{x}=\partial / \partial_{x}$ and so on. Using Eq. (2.3) in Eq. (2.1) the equations of motion are written

$$
\nabla_{i J} c_{J K} \nabla_{K l} u_{l}=\rho \partial_{t}^{2} u_{i}
$$

\section{SERIES EXPANSIONS}

In order to derive approximate plate equations for the anisotropic plate, the displacement components are now expanded in power series in the thickness coordinate according to

$$
u_{i}(x, y, z, t)=\sum_{n=0}^{\infty} z^{n} u_{i}^{(n)}(x, y, t)
$$

where $\left[u_{i}^{(n)}\right]=\left(\begin{array}{llll}u_{n} & v_{n} & w_{n}\end{array}\right)^{T}$ are expansion functions independent of $z$. Note that these expansions involve both even and odd powers of $z$ as symmetric and antisymmetric motions with respect to $z$ do not decouple in the general case. Inserting the series expansions Eq. (3.1) into the equations of motion Eq. (2.5) and collecting equal powers of $z$, the solution of the equation system for each power yields the recursion formulas

$$
\begin{aligned}
u_{i}^{(n+2)}=\frac{1}{(n+1)(n+2)} M_{i j} & {\left[\rho \ddot{u}_{j}^{(n)}-\bar{\nabla}_{j K} c_{K L} \bar{\nabla}_{L m} u_{m}^{(n)}\right.} \\
& \left.-(n+1)\left(L_{j K} c_{K L} \bar{\nabla}_{L m}+\bar{\nabla}_{j K} c_{K L} L_{L m}\right) u_{m}^{(n+1)}\right] .
\end{aligned}
$$


Here, $\left[M_{i j}\right]=\left[C_{i j}\right]^{-1}$ and $\left[L_{I j}\right]=\left[L_{i J}\right]^{T}$ where

$$
\left[C_{i j}\right]=\left(\begin{array}{ccc}
c_{55} & c_{45} & c_{35} \\
c_{45} & c_{44} & c_{34} \\
c_{35} & c_{34} & c_{33}
\end{array}\right), \quad\left[L_{i J}\right]=\left(\begin{array}{cccccc}
0 & 0 & 0 & 0 & 1 & 0 \\
0 & 0 & 0 & 1 & 0 & 0 \\
0 & 0 & 1 & 0 & 0 & 0
\end{array}\right)
$$

The matrix operators with an overline $\left(\bar{\nabla}_{i J}\right.$ and $\left.\bar{\nabla}_{I j}\right)$ are introduced for convenience through

$$
\nabla_{i J}=\bar{\nabla}_{i J}+\partial_{z} L_{i J}, \quad \nabla_{I j}=\bar{\nabla}_{I j}+\partial_{z} L_{I j}
$$

Hence, they are the same as $\nabla_{i J}$ and $\nabla_{I j}$ in Eq. (2.4) but with all derivatives with respect to $z$ put to zero, i.e. $\partial_{z}=0$.

The recursion relations can be used to express all expansion functions $u_{i}^{(n)}$ with $n=$ $\{2,3, \ldots\}$ in terms of the lowest-order ones with $n=0,1$. This turns the recursion relations Eq. (3.2) into

$$
u_{i}^{(n)}=\frac{1}{n !}\left[A_{i j}^{(n-2)} u_{j}^{(0)}+B_{i j}^{(n-2)} u_{j}^{(1)}\right], \quad n=0,1,2, \ldots .
$$

Here the differential operators $A_{i j}^{(n)}$ and $B_{i j}^{(n)}$ are defined recursively as

$$
\begin{array}{ll}
A_{i k}^{(n)}=B_{i j}^{(0)} A_{j k}^{(n-1)}+A_{i j}^{(0)} A_{j k}^{(n-2)}, & n=0,1,2, \ldots, \\
B_{i k}^{(n)}=B_{i j}^{(0)} B_{j k}^{(n-1)}+A_{i j}^{(0)} B_{j k}^{(n-2)}, & n=0,1,2, \ldots,
\end{array}
$$

where

$$
\begin{array}{lll}
A_{i j}^{(-2)}=\delta_{i j}, & A_{i j}^{(-1)}=0, & A_{i m}^{(0)}=M_{i j}\left(\delta_{j m} \rho \partial_{t}^{2}-\bar{\nabla}_{j K} c_{K L} \bar{\nabla}_{L m}\right), \\
B_{i j}^{(-2)}=0, & B_{i j}^{(-1)}=\delta_{i j}, & B_{i m}^{(0)}=-M_{i j}\left(L_{j K} c_{K L} \bar{\nabla}_{L m}+\bar{\nabla}_{j K} c_{K L} L_{L m}\right),
\end{array}
$$

using $\left[\delta_{i j}\right]$ as the identity matrix. Note that these recursion formulas involve no approximations since they stem from the definition of the series expansions (3.1) and are as such exact. Moreover, no truncations of the displacement terms have so far been performed, which is of crucial importance for the present method.

Using the expansion ansatz Eq. (3.1) in the stresses Eq. (2.3) results in

$$
T_{I}=\sum_{n=0}^{\infty} z^{n} T_{I}^{(n)}
$$

where

$$
T_{I}^{(n)}=c_{I J}\left[\bar{\nabla}_{J k} u_{k}^{(n)}+(n+1) L_{J k} u_{k}^{(n+1)}\right] .
$$

Hereby the stresses may be expressed in the lowest-order terms adopting Eq. (3.5)

$$
T_{I}^{(n)}=\frac{c_{I J}}{n !}\left[\left(\bar{\nabla}_{J k} A_{k l}^{(n-2)}+L_{J k} A_{k l}^{(n-1)}\right) u_{l}^{(0)}+\left(\bar{\nabla}_{J k} B_{k l}^{(n-2)}+L_{J k} B_{k l}^{(n-1)}\right) u_{l}^{(1)}\right] .
$$




\section{Plate equations}

At each point on the surfaces $z= \pm h$, either the traction or the displacement is to be prescribed in each coordinate direction. These given fields are denoted by $\left\{T_{I}^{+}, u_{i}^{+}\right\}$and $\left\{T_{I}^{-}, u_{i}^{-}\right\}$at the upper and lower surfaces, respectively, where $I=5,4,3$ and $i=x, y, z$. Hence, one of the fields for each of the six pairs $\left\{T_{x z}^{ \pm}, u^{ \pm}\right\},\left\{T_{y z}^{ \pm}, v^{ \pm}\right\},\left\{T_{z z}^{ \pm}, w^{ \pm}\right\}$is to be given, resulting in three boundary conditions at each point on the upper and lower surfaces, respectively. For prescribed tractions, the boundary conditions adopting Eq. (3.8) to order $N$ become

$$
\sum_{n=0}^{N} h^{n} T_{I}^{(n)}(x, y, t)=T_{I}^{+}(x, y, t), \quad \sum_{n=0}^{N}(-h)^{n} T_{I}^{(n)}(x, y, t)=T_{I}^{-}(x, y, t),
$$

for $I=5,4,3$. Similarly, the boundary conditions for prescribed displacements adopting Eq. (3.1) to order $N+1$ become

$$
\sum_{n=0}^{N+1} h^{n} u_{i}^{(n)}(x, y, t)=u_{i}^{+}(x, y, t), \quad \sum_{n=0}^{N+1}(-h)^{n} u_{i}^{(n)}(x, y, t)=u_{i}^{-}(x, y, t)
$$

for $i=x, y, z$. The difference in number of terms used for Eq. (4.1) and Eq. (4.2) is due to the differences in the differential orders. Since the stress terms $T_{I}^{(n)}$ include spatial derivatives of one order higher than the corresponding displacements $u_{i}^{(n)}$, an extra term is to be included in the latter sums to obtain a consistent set of plate equations.

The surface boundary conditions obtained from Eqs. (4.1)-(4.2) constitute the hyperbolic set of six scalar plate equations. Using Eqs. (3.5) and (3.10), these plate equations may be expressed in terms of the six lowest-order displacement fields $u_{i}^{(n)}$ for $n=0,1$. Depending on whether traction or displacement based boundary conditions are adopted on a surface region, the governing set of differential equations are in the general case fundamentally different for various parts of the plate. It could be noted here that these boundary conditions are in all cases fulfilled exactly for the expansion order in question. Each of the six boundary conditions involves spatial derivatives of order $N+1$ on $u_{i}^{(0)}$ (except when $N=0$ for prescribed displacements) and spatial derivatives of order $N$ on $u_{i}^{(1)}$. However, the resulting system of six plate equations turns out to be of differential order $2(3 N-1)$ in the $x y$ coordinates due to cancelation effects. This is readily seen by eliminating all but one of the fields, say $w_{0}$, resulting in one equation of spatial order $2(3 N-1)$. Consequently, there are to be stated $3 N-1$ boundary conditions along the edge of the plate.

\section{EDGE BOUNDARY CONDITIONS}

Consider a rectangular plate where $-a \leq x \leq a$ and $-b \leq y \leq b$. As for the surfaces $z= \pm h$, either the traction or the displacement is to be prescribed in each coordinate direction. At $x= \pm a$ these fields are denoted $\left\{T_{I}^{ \pm a}, u_{i}^{ \pm a}\right\}$ where $I=1,6,5$ and $i=x, y, z$. Consequently, one of the quantities for each of the pairs $\left\{T_{x x}^{ \pm a}, u^{ \pm a}\right\},\left\{T_{x y}^{ \pm a}, v^{ \pm a}\right\},\left\{T_{x z}^{ \pm a}, w^{ \pm a}\right\}$ is to be given at each edge point for $x= \pm a$. Analogously, the prescribed fields at $y= \pm b$ are denoted $\left\{T_{I}^{ \pm b}, u_{i}^{ \pm b}\right\}$ where $I=6,2,4$ and $i=x, y, z$. Hereby one of the quantities for each of the six pairs $\left\{T_{x y}^{ \pm b}, u^{ \pm b}\right\},\left\{T_{y y}^{ \pm b}, v^{ \pm b}\right\},\left\{T_{y z}^{ \pm b}, w^{ \pm b}\right\}$ is given at each edge point for $y= \pm b$. 
The edge boundary conditions may be obtained by adopting variational techniques. Here a generalized Hamilton's principle is used where displacements and stresses are varied simultaneously and independently [27]. This has the effect that both displacement and traction based boundary conditions are treated in the same rigorous fashion. Assume from now on that either a displacement component or the corresponding traction component is prescribed over the entire thickness interval $-h \leq z \leq h$ for each $\{x, y\}$ at the boundaries. This is generally the case when studying problems addressed by approximate plate theories. For the more involved situation where this restriction is lifted, such problems must probably be solved using plate theories of quite high order to give reliable results [27].

Since all boundary fields are derived in the same manner, the procedure is presented for a given normal traction at $x=a$. Assume the prescribed stress $T_{x x}^{+a}(y, z, t)$. Following the results from variational calculus presented in [27], the edge boundary conditions are obtained from

$$
\int_{-h}^{h}\left(T_{x x}^{+a}(y, z, t)-\sum_{n=0}^{M} z^{n} T_{x x}^{(n)}(a, y, t)\right) z^{k} d z=0, \quad k=0,1, \ldots, M,
$$

adopting Eq. (3.8) to order $M$. It is thus straightforward to derive the $M+1$ unknowns $T_{x x}^{(n)}$ from the system of $M+1$ equations. It is readily seen that the hereby obtained representation of the boundary stress function $T_{x x}$ in power series is actually identical to the expansion of the given function $T_{x x}^{+a}$ in terms of Legendre polynomials $P_{k}(z / h)$ of order $M$. Note that the standard case $T_{x x}^{+a}=0$ results in $T_{x x}^{(n)}=0$ for all $n$.

In line with the derivative order of the system discussed above for the boundary conditions at the upper and lower surfaces, the number of edge boundary conditions at each edge is $3 N-1$. For each point $y$ at $x= \pm a$, these are distributed among the fields as $N$ terms for $\left\{T_{x x}^{(n)}, u_{n}\right\}, N$ terms for $\left\{T_{x y}^{(n)}, v_{n}\right\}$, and $N-1$ terms for $\left\{T_{x z}^{(n)}, w_{n}\right\}$. Here either the stress or the displacement field is to be used for each pair, depending on the formulation of the prescribed boundary conditions. Note that the first terms are for $n=0$. Similarly, edge boundary conditions at $y= \pm b$ are according to $N$ terms for $\left\{T_{x y}^{(n)}, u_{n}\right\}, N$ terms for $\left\{T_{y y}^{(n)}, v_{n}\right\}$, and $N-1$ terms for $\left\{T_{y z}^{(n)}, w_{n}\right\}$ in each case. Consequently, the boundary conditions for stresses involve one order higher spatial derivative than the corresponding boundary conditions for displacements. The lower number of terms for the fields directed in the $z$ direction is in line with the corresponding results from [27]. In the present fully anisotropic case this feature may not be explained in an equally straightforward manner. However, this behavior becomes clearer when studying an orthotropic plate, see Section 6.2

5.1. Coupling conditions. When homogeneous rectangular plates with different properties (geometrical, material, surface boundary conditions) are joined together, there are $2(3 N-1)$ coupling conditions to be fulfilled along a common edge. For coupling along the boundaries $x= \pm a$, there should be $N$ conditions on $u, v, T_{x x}, T_{x y}$ as well as $N-1$ conditions on $w$ and $T_{x z}$ at each edge for every point $y$. Naturally, similar conditions hold for coupling along the boundaries $y= \pm b$ for every point $x$. Consider first the normal displacement and stress fields for coupling at $x= \pm a$, using the series expansion approach Eqs. (3.1) and (3.8). Following the procedure in [27], the displacement conditions result 
in termwise equality $u_{n}^{l}=u_{n}^{r}$. Here the superscript " $l$ " is for the left-hand fields and " $r$ " is for the right-hand fields. For the normal stress, Eq. (5.1) is extended to

$$
\int_{h^{l}} \sum_{n=0}^{N-1} z^{n} T_{x x}^{(n)}\left(a^{l}, y, t\right) z^{k} d z=\int_{h^{r}} \sum_{n=0}^{N-1} z^{n} T_{x x}^{(n)}\left(a^{r}, y, t\right) z^{k} d z, \quad k=0,1, \ldots, N-1,
$$

where the integrals are taken over each plate thickness, respectively. This stress condition is a generalized force continuity requirement. In the special case $h^{l}=h^{r}$ each stress term is equal on both sides. The same procedure holds for the other fields, as well as at the boundaries $y= \pm b$.

When calculating displacements and stresses anywhere in a plate, the number of terms used in the series representations could be chosen in several ways. One possibility is to consistently adopt the same number of terms as in the edge conditions, that is $N$ terms for the fields $u, v, T_{x x}, T_{y y}, T_{z z}, T_{x y}$ and $N-1$ terms for the fields $w, T_{x z}, T_{y z}$. Another possibility is to use the same number of fields as in the surface boundary conditions Eqs. (4.1)- (4.2), that is $N+2$ terms for the displacements and $N+1$ terms for the stresses. Based on experiences from [27], the latter approach is used here. This causes the upper and lower surface boundary conditions to be fulfilled exactly, but corrupts the edge conditions.

\section{Orthotropic plate}

The plate equations described above for fully anisotropic plates may be used to derive explicit equations for the special case of orthotropic plates. Such material configurations are of special importance in many engineering applications. For crystal axes along the coordinate axes, the stiffness matrix reduces to

$$
\left[c_{I J}\right]=\left(\begin{array}{cccccc}
c_{11} & c_{12} & c_{13} & 0 & 0 & 0 \\
c_{12} & c_{22} & c_{23} & 0 & 0 & 0 \\
c_{13} & c_{23} & c_{33} & 0 & 0 & 0 \\
0 & 0 & 0 & c_{44} & 0 & 0 \\
0 & 0 & 0 & 0 & c_{55} & 0 \\
0 & 0 & 0 & 0 & 0 & c_{66}
\end{array}\right)
$$

involving nine independent stiffness constants. From Eq. (3.2) the recursion formulas become

$$
\begin{aligned}
u_{n+2} & =\frac{1}{(n+1)(n+2) c_{55}}\left[\rho \partial_{t}^{2} u_{n}-c_{11} \partial_{x}^{2} u_{n}-c_{66} \partial_{y}^{2} u_{n}\right. \\
& \left.-c_{126} \partial_{x} \partial_{y} v_{n}-(n+1) c_{135} \partial_{x} w_{n+1}\right], \\
v_{n+2} & =\frac{1}{(n+1)(n+2) c_{44}}\left[\rho \partial_{t}^{2} v_{n}-c_{66} \partial_{x}^{2} v_{n}-c_{22} \partial_{y}^{2} v_{n}\right. \\
& \left.-c_{126} \partial_{x} \partial_{y} u_{n}-(n+1) c_{234} \partial_{y} w_{n+1}\right], \\
w_{n+2} & =\frac{1}{(n+1)(n+2) c_{33}}\left[\rho \partial_{t}^{2} w_{n}-c_{55} \partial_{x}^{2} w_{n}-c_{44} \partial_{y}^{2} w_{n}\right. \\
& \left.-(n+1) c_{135} \partial_{x} u_{n+1}-(n+1) c_{234} \partial_{y} v_{n+1}\right],
\end{aligned}
$$


where $n=0,1,2, \ldots$ Here the new stiffness constants are defined as (no summation convention) $c_{i j k}=c_{i j}+c_{k k}$. Similarly, the expanded stresses according to Eq. (3.9) are written

$$
\begin{aligned}
& T_{x x}^{(n)}=c_{11} \partial_{x} u_{n}+c_{12} \partial_{y} v_{n}+(n+1) c_{13} w_{n+1}, \\
& T_{y y}^{(n)}=c_{12} \partial_{x} u_{n}+c_{22} \partial_{y} v_{n}+(n+1) c_{23} w_{n+1}, \\
& T_{z z}^{(n)}=c_{13} \partial_{x} u_{n}+c_{23} \partial_{y} v_{n}+(n+1) c_{33} w_{n+1}, \\
& T_{x z}^{(n)}=c_{55}\left[(n+1) u_{n+1}+\partial_{x} w_{n}\right], \\
& T_{y z}^{(n)}=c_{44}\left[(n+1) v_{n+1}+\partial_{y} w_{n}\right], \\
& T_{x y}^{(n)}=c_{66}\left(\partial_{y} u_{n}+\partial_{x} v_{n}\right),
\end{aligned}
$$

for $n=0,1,2, \ldots$. By adopting the recursion formulas repeatedly, the expanded displacement and stress fields are expressed in terms of the lowest-order fields $u_{i}^{(n)}$ for $n=0,1$ as in Eqs. (3.5) and (3.10). By inserting these expressions into the equations presented in Sections $4-5$, six coupled orthotropic plate equations with pertinent edge boundary conditions are hereby obtained written in terms of the lowest-order fields $u_{i}^{(n)}$ for $n=0,1$.

6.1. Plate equations. Consider for simplicity the case when either the traction or the displacement is prescribed on both the upper and lower surfaces for a specific direction. By adding and subtracting the boundary conditions in Eqs. (4.1)-(4.2), equations containing only even or odd expansions in $h$ are obtained according to

$$
\begin{aligned}
\sum_{n=0}^{\lfloor N / 2\rfloor} h^{2 n} T_{I}^{(2 n)} & =\frac{1}{2}\left(T_{I}^{+}+T_{I}^{-}\right), & \sum_{n=0}^{\lfloor(N-1) / 2\rfloor} h^{2 n+1} T_{I}^{(2 n+1)} & =\frac{1}{2}\left(T_{I}^{+}-T_{I}^{-}\right), \\
\sum_{n=0}^{\lfloor(N+1) / 2\rfloor} h^{2 n} u_{i}^{(2 n)} & =\frac{1}{2}\left(u_{i}^{+}+u_{i}^{-}\right), & \sum_{n=0}^{\lfloor N / 2\rfloor} h^{2 n+1} u_{i}^{(2 n+1)} & =\frac{1}{2}\left(u_{i}^{+}-u_{i}^{-}\right),
\end{aligned}
$$

where $I=5,4,3$ and $i=x, y, z$. Here, the stress and displacement expansion functions $T_{I}^{(n)}$ and $u_{i}^{(n)}$ are given by Eqs. (3.10) and (3.5), while $\lfloor n\rfloor$ denotes the floor of $n$, i.e. the nearest lower integer to $n$. These equations constitute decoupled sets corresponding to symmetrical and antisymmetrical motions. Considering the three stress components in Eq. (6.6), symmetric motion is obtained by taking $I=3$ in the first relation and $I=5,4$ in the second relation. Similarly, a symmetric set is obtained for the three displacement components in Eq. (6.7) by taking $i=x, y$ in the first relation and $i=z$ in the second relation. For all possible combinations of such symmetric boundary conditions, the equations involve only the fields $u_{0}, v_{0}, w_{1}$. Naturally the complementary combinations correspond to antisymmetrical sets involving $u_{1}, v_{1}, w_{0}$. It should be noted that odd order $N$ are mainly of interest here. This is readily seen by eliminating the fields to one equation, involving every second power in $h$. Hereby, the time and spatial derivatives in the resulting equation are asymptotically correct up to and including order $N+1$. For $N$ even, only the derivatives of order $N$ are correctly represented.

For the sake of clarity, the plate equations of order $N=3$ are presented explicitly below in the traditional case when the traction is prescribed on both surfaces. The main reason to use this order is that flexural effects are hereby correctly accounted for through the antisymmetric $h^{3}$ terms. For the antisymmetric motion the three plate equations become 


$$
\begin{aligned}
& c_{55}\left(u_{1}+\partial_{x} w_{0}\right)+\frac{h^{2}}{2}\left[\rho \partial_{t}^{2} u_{1}+\left(\beta_{133} c_{135}-c_{11}\right) \partial_{x}^{2} u_{1}-c_{66} \partial_{y}^{2} u_{1}\right. \\
& \left.\left(\beta_{133} c_{234}-c_{126}\right) \partial_{x} \partial_{y} v_{1}-\beta_{133} \rho \partial_{t}^{2} \partial_{x} w_{0}+\beta_{133} c_{55} \partial_{x}^{3} w_{0}+\beta_{133} c_{44} \partial_{x} \partial_{y}^{2} w_{0}\right]=\frac{1}{2}\left(T_{x z}^{+}+T_{x z}^{-}\right), \\
& c_{44}\left(v_{1}+\partial_{y} w_{0}\right)+\frac{h^{2}}{2}\left[\rho \partial_{t}^{2} v_{1}+\left(\beta_{233} c_{234}-c_{22}\right) \partial_{y}^{2} v_{1}-c_{66} \partial_{x}^{2} v_{1}\right. \\
& \left.+\left(\beta_{233} c_{135}-c_{126}\right) \partial_{x} \partial_{y} u_{1}-\beta_{233} \rho \partial_{t}^{2} \partial_{y} w_{0}+\beta_{233} c_{44} \partial_{y}^{3} w_{0}+\beta_{233} c_{55} \partial_{x}^{2} \partial_{y} w_{0}\right]=\frac{1}{2}\left(T_{y z}^{+}+T_{y z}^{-}\right),
\end{aligned}
$$

$$
\begin{aligned}
& h\left[\rho \partial_{t}^{2} w_{0}-c_{55}\left(\partial_{x} u_{1}+\partial_{x}^{2} w_{0}\right)-c_{44}\left(\partial_{y} v_{1}+\partial_{y}^{2} w_{0}\right)\right] \\
& +\frac{h^{3}}{6 c_{33}}\left[\rho^{2} \partial_{t}^{4} w_{0}-c_{13} c_{55} \partial_{x}^{4} w_{0}-c_{23} c_{44} \partial_{y}^{4} w_{0}-\left(c_{55}-c_{13}\right) \rho \partial_{t}^{2} \partial_{x}^{2} w_{0}\right. \\
& -\left(c_{44}-c_{23}\right) \rho \partial_{t}^{2} \partial_{y}^{2} w_{0}-\left(c_{13} c_{44}+c_{23} c_{55}\right) \partial_{x}^{2} \partial_{y}^{2} w_{0} \\
& -\left(c_{33}+c_{135}\right) \rho \partial_{t}^{2} \partial_{x} u_{1}-\left(c_{33}+c_{234}\right) \rho \partial_{t}^{2} \partial_{y} v_{1}+\left(c_{11} c_{33}-c_{13} c_{135}\right) \partial_{x}^{3} u_{1}+\left(c_{22} c_{33}-c_{23} c_{234}\right) \partial_{y}^{3} v_{1} \\
& \left.+\left[c_{33}\left(c_{126}+c_{66}\right)-c_{23} c_{135}\right] \partial_{x} \partial_{y}^{2} u_{1}+\left[c_{33}\left(c_{126}+c_{66}\right)-c_{13} c_{234}\right] \partial_{x}^{2} \partial_{y} v_{1}\right]=\frac{1}{2}\left(T_{z z}^{+}-T_{z z}^{-}\right) .
\end{aligned}
$$

Here the new constants are defined as (no summation convention) $\beta_{i j k}=c_{i j} / c_{k k}$. Note that the specialized case of an isotropic plate renders identical equations to those presented by Boström et al. [20].

Similarly, the three plate equations for the symmetric motion are

$$
\begin{aligned}
& h\left(\rho \partial_{t}^{2} u_{0}-c_{11} \partial_{x}^{2} u_{0}-c_{66} \partial_{y}^{2} u_{0}-c_{126} \partial_{x} \partial_{y} v_{0}-c_{13} \partial_{x} w_{1}\right) \\
& +\frac{h^{3}}{6 c_{55}}\left[\rho^{2} \partial_{t}^{4} u_{0}+c_{11} c_{55} \alpha_{11} \partial_{x}^{4} u_{0}+c_{66}^{2} \partial_{y}^{4} u_{0}-c_{55}\left(\alpha_{11}+\beta_{115}\right) \rho \partial_{t}^{2} \partial_{x}^{2} u_{0}\right. \\
& -2 c_{66} \rho \partial_{t}^{2} \partial_{y}^{2} u_{0}+c_{55}\left[c_{12} \alpha_{12}+c_{66}\left(\alpha_{11}+\alpha_{12}+\alpha_{4}+\beta_{115}\right)\right] \partial_{x}^{2} \partial_{y}^{2} u_{0} \\
& -c_{55}\left(\alpha_{12}+\alpha_{5}+\beta_{664}\right) \rho \partial_{t}^{2} \partial_{x} \partial_{y} v_{0}+c_{55}\left[c_{12} \alpha_{11}+c_{66}\left(\alpha_{11}+\alpha_{12}+\beta_{664}\right)\right] \partial_{x}^{3} \partial_{y} v_{0} \\
& +c_{55}\left[c_{22} \alpha_{12}+c_{66}\left(\alpha_{5}+\beta_{224}\right)\right] \partial_{x} \partial_{y}^{3} v_{0}-c_{55} \alpha_{1} \rho \partial_{t}^{2} \partial_{x} w_{1}+c_{55}\left[c_{11}+c_{13}\left(\alpha_{11}-\beta_{133}\right)\right] \partial_{x}^{3} w_{1} \\
& \left.+c_{55}\left[c_{12}+c_{23}\left(\alpha_{12}-\beta_{133}\right)+c_{66} \alpha_{3}\right] \partial_{x} \partial_{y}^{2} w_{1}\right]=\frac{1}{2}\left(T_{x z}^{+}-T_{x z}^{-}\right),
\end{aligned}
$$




$$
\begin{aligned}
& h\left(\rho \partial_{t}^{2} v_{0}-c_{22} \partial_{y}^{2} v_{0}-c_{66} \partial_{x}^{2} v_{0}-c_{126} \partial_{x} \partial_{y} u_{0}-c_{23} \partial_{y} w_{1}\right) \\
& +\frac{h^{3}}{6 c_{44}}\left[\rho^{2} \partial_{t}^{4} v_{0}+c_{22} c_{44} \alpha_{22} \partial_{y}^{4} v_{0}+c_{66}^{2} \partial_{x}^{4} v_{0}-c_{44}\left(\alpha_{22}+\beta_{224}\right) \rho \partial_{t}^{2} \partial_{y}^{2} v_{0}\right. \\
& -2 c_{66} \rho \partial_{t}^{2} \partial_{x}^{2} v_{0}+c_{44}\left[c_{12} \alpha_{21}+c_{66}\left(\alpha_{22}+\alpha_{21}+\alpha_{5}+\beta_{224}\right)\right] \partial_{x}^{2} \partial_{y}^{2} v_{0} \\
& -c_{44}\left(\alpha_{21}+\alpha_{4}+\beta_{665}\right) \rho \partial_{t}^{2} \partial_{x} \partial_{y} u_{0}+c_{44}\left[c_{12} \alpha_{22}+c_{66}\left(\alpha_{22}+\alpha_{21}+\beta_{665}\right)\right] \partial_{x} \partial_{y}^{3} u_{0} \\
& +c_{44}\left[c_{11} \alpha_{21}+c_{66}\left(\alpha_{4}+\beta_{115}\right)\right] \partial_{x}^{3} \partial_{y} u_{0}-c_{44} \alpha_{2} \rho \partial_{t}^{2} \partial_{y} w_{1}+c_{44}\left[c_{22}+c_{23}\left(\alpha_{22}-\beta_{233}\right)\right] \partial_{y}^{3} w_{1} \\
& \left.+c_{44}\left[c_{12}+c_{13}\left(\alpha_{21}-\beta_{233}\right)+c_{66} \alpha_{3}\right] \partial_{x}^{2} \partial_{y} w_{1}\right]=\frac{1}{2}\left(T_{y z}^{+}-T_{y z}^{-}\right) \\
& c_{33} w_{1}+c_{13} \partial_{x} u_{0}+c_{23} \partial_{y} v_{0}+\frac{h^{2}}{2}\left[\rho \partial_{t}^{2} w_{1}+c_{13} \partial_{x}^{2} w_{1}+c_{23} \partial_{y}^{2} w_{1}\right. \\
& -\rho \partial_{t}^{2} \partial_{x} u_{0}+c_{11} \partial_{x}^{3} u_{0}+\left(c_{12}+2 c_{66}\right) \partial_{x} \partial_{y}^{2} u_{0} \\
& \left.-\rho \partial_{t}^{2} \partial_{y} v_{0}+c_{22} \partial_{y}^{3} v_{0}+\left(c_{12}+2 c_{66}\right) \partial_{x}^{2} \partial_{y} v_{0}\right]=\frac{1}{2}\left(T_{z z}^{+}+T_{z z}^{-}\right) .
\end{aligned}
$$

Here some additional constants are introduced according to

$$
\begin{array}{ll}
\alpha_{11}=\beta_{115}-\beta_{133}-\beta_{133} \beta_{135}, & \alpha_{12}=\beta_{124}-\beta_{133}-\beta_{133} \beta_{234}, \\
\alpha_{22}=\beta_{224}-\beta_{233}-\beta_{233} \beta_{234}, & \alpha_{21}=\beta_{125}-\beta_{233}-\beta_{233} \beta_{135}, \\
\alpha_{1}=1+\beta_{133}+\beta_{135}, & \alpha_{2}=1+\beta_{233}+\beta_{234}, \quad \alpha_{3}=2+\beta_{234}+\beta_{135} \\
\alpha_{4}=\beta_{124}+\beta_{664}, & \alpha_{5}=\beta_{125}+\beta_{665},
\end{array}
$$

Specializing to an isotropic plate, the equations given in Johansson et al. [32] may be retrieved. Note that when deleting the $h^{3}$ and $h^{2}$ terms $(N=1)$ this set corresponds to the generalized plane stress case.

Using these six equations, $\mathrm{P} / \mathrm{SV}$ and $\mathrm{SH}$ waves couple in the general orthotropic case. However, for a transversely isotropic material (five independent stiffness constants) with $x y$ as the plane of isotropy, the $\mathrm{P} / \mathrm{SV}$ and the $\mathrm{SH}$ waves decouple. Naturally, the same holds for an isotropic material (two independent stiffness constants). These coupling effects have to be taken into consideration when specializing from orthotropic to transversely isotropic materials, especially if fields are to be eliminated. As an example, it is possible to eliminate $u_{1}$ and $v_{1}$ in the antisymmetric case, Eqs. (6.8)-(6.10), to get one single equation in only $w_{0}$. This equation includes $h^{7}$ terms that may be truncated to order $h^{3}$. However, this truncated equation can not properly deal with the simpler specialized case of transverse isotropy. The explanation is the coupling between the $\mathrm{P} / \mathrm{SV}$ and the $\mathrm{SH}$ wave in an orthotropic material, that does not appear in a transversely isotropic material. In the latter case, the original equation of order $h^{7}$ can be factorized into two equations describing the $\mathrm{P} / \mathrm{SV}$ wave and $\mathrm{SH}$ wave, respectively. Hence, if truncations are made before the specialization to transverse isotropy, the factorization is not possible and the single equation truncated to order $h^{3}$ (with contributions from both $\mathrm{P} / \mathrm{SV}$ and $\mathrm{SH}$ ) is of course something different from the correctly truncated equation of order $h^{3}$ (with contributions from only $\mathrm{P} / \mathrm{SV}$ ). Consequently, the set of six plate equations are generally to be maintained without elimination of the fields within. This is especially the case when edge boundary conditions are considered as these are expressed in terms of the six fields, see below. 
6.2. Edge boundary conditions. Consider the standard situation when the plate is either fixed $u_{i}^{ \pm\{a, b\}}=0$, or free $T_{I}^{ \pm\{a, b\}}=0$ in each coordinate direction, respectively. Consequently, either $u_{i}^{(n)}=0$ or $T_{I}^{(n)}=0$ at each edge for the orders $n$ in question. Among the total number of $3 N-1$ edge boundary conditions at each edge, $3\lfloor N / 2\rfloor$ conditions are for the antisymmetric motion. These conditions are shared equally among the fields in the three directions, which in the clamped case results in $u_{2 k+1}=0, v_{2 k+1}=0$ and $w_{2 k}=0$ for $k=0,1 \ldots\lfloor(N-2) / 2\rfloor$. Similarly, the remaining symmetric boundary conditions are $u_{2 k}=0, v_{2 k}=0$ and $w_{2 k-1}=0$ for $k=0,1 \ldots\lfloor(N-1) / 2\rfloor$. Consequently, there are one term less in the $z$ direction than in the $x$ and $y$ directions for the symmetric motion, which is in line with the general discussion in Section 5.

These boundary conditions are given explicitly below for $N=3$. For the antisymmetric case, there are three boundary conditions to be fulfilled at each edge. At the edges $x= \pm a$ these conditions read

$$
\begin{aligned}
& u_{1}=0 \quad \text { or } \quad T_{x x}^{(1)}=\left(c_{11}-\beta_{133} c_{135}\right) \partial_{x} u_{1}+\left(c_{12}-\beta_{133} c_{234}\right) \partial_{y} v_{1} \\
& +\beta_{133} \rho \partial_{t}^{2} w_{0}-\beta_{133} c_{55} \partial_{x}^{2} w_{0}-\beta_{133} c_{44} \partial_{y}^{2} w_{0}=0, \\
& v_{1}=0 \quad \text { or } \quad T_{x y}^{(1)}=c_{66}\left(\partial_{y} u_{1}+\partial_{x} v_{1}\right)=0 \text {, } \\
& w_{0}=0 \quad \text { or } \quad T_{x z}^{(0)}=c_{55}\left(u_{1}+\partial_{x} w_{0}\right)=0 .
\end{aligned}
$$

The physical interpretation of these boundary conditions is that either the angle or the bending moment $M_{x}$ is zero, either the rotation or the torsion $M_{x y}$ is zero, and either the vertical displacement or the shear force $T_{z}$ is zero, respectively.

Similar results are obtained at the edges $y= \pm b$ according to

$$
\begin{aligned}
& v_{1}=0 \quad \text { or } \quad T_{y y}^{(1)}=\left(c_{22}-\beta_{233} c_{234}\right) \partial_{y} v_{1}+\left(c_{12}-\beta_{233} c_{135}\right) \partial_{x} u_{1} \\
& +\beta_{233} \rho \partial_{t}^{2} w_{0}-\beta_{233} c_{55} \partial_{x}^{2} w_{0}-\beta_{233} c_{44} \partial_{y}^{2} w_{0}=0 . \\
& u_{1}=0 \quad \text { or } \quad T_{x y}^{(1)}=c_{66}\left(\partial_{y} u_{1}+\partial_{x} v_{1}\right)=0 \text {, } \\
& w_{0}=0 \quad \text { or } \quad T_{y z}^{(0)}=c_{44}\left(v_{1}+\partial_{y} w_{0}\right)=0 \text {, }
\end{aligned}
$$

with analogous interpretations as above.

For the symmetric case, there are five boundary conditions to be fulfilled at each edge. At the edges $x= \pm a$ these conditions become

$$
\begin{aligned}
& u_{0}=0, \quad u_{2}=0 \quad \text { or } \\
& T_{x x}^{(0)}=c_{11} \partial_{x} u_{0}+c_{12} \partial_{y} v_{0}+c_{13} w_{1}=0, \quad T_{x x}^{(2)}=0, \\
& v_{0}=0, \quad v_{2}=0 \quad \text { or } \quad \text { or } \\
& T_{x y}^{(0)}=c_{66}\left(\partial_{y} u_{0}+\partial_{x} v_{0}\right)=0, \quad T_{x y}^{(2)}=0, \\
& w_{1}=0 \quad c_{x z}^{(1)}=\rho \partial_{t}^{2} u_{0}-c_{11} \partial_{x}^{2} u_{0}-c_{66} \partial_{y}^{2} u_{0}-\left(c_{12}+c_{66}\right) \partial_{x} \partial_{y} v_{0}-c_{13} \partial_{x} w_{1}=0 .
\end{aligned}
$$

Here and below the fields $u_{i}^{(2)}=0$ and $T_{I}^{(2)}=0$ are not given explicitly for brevity. Using Eq. (6.5) together with the recursion formulas Eq. (6.2)-(6.4) these fields are readily expressed in terms of $\left\{u_{0}, v_{0}, w_{1}\right\}$. The physical interpretation of these boundary conditions is that either the normal horizontal displacement or the normal force $N_{x}$ is zero, 
either the tangential horizontal displacement or the shear force $T_{y}$ is zero, and either the vertical stretching or the stretch force is zero.

The results at the edges $y= \pm b$ are

$$
\begin{aligned}
& v_{0}=0, \quad v_{2}=0 \quad \text { or } \\
& T_{y y}^{(0)}=c_{22} \partial_{y} v_{0}+c_{12} \partial_{x} u_{0}+c_{23} w_{1}=0, \quad T_{y y}^{(2)}=0, \\
& u_{0}=0, \quad u_{2}=0 \quad \text { or } \\
& T_{x y}^{(0)}=c_{66}\left(\partial_{y} u_{0}+\partial_{x} v_{0}\right)=0, \quad T_{x y}^{(2)}=0, \\
& w_{1}=0 \quad \text { or } \\
& T_{y z}^{(1)}=\rho \partial_{t}^{2} v_{0}-c_{22} \partial_{y}^{2} v_{0}-c_{66} \partial_{x}^{2} v_{0}-\left(c_{12}+c_{66}\right) \partial_{x} \partial_{y} u_{0}-c_{23} \partial_{y} w_{1}=0 .
\end{aligned}
$$

Note that in many engineering applications, the antisymmetric bending effects are much more important than the symmetric effects. Due to this statement, there are prospects to handle the symmetrical motion using a simpler set of equations. Hence, one such simplification is to involve symmetrical equations of order $N=1$ together with antisymmetrical equations of order $N=3$.

\section{Analytical COMparison to other approximate theOries}

This section aims at comparing the present theory of order $N=3$ to other approximate theories in the case of antisymmetric motion of an orthotropic plate. The comparisons are made with respect to both the equations of motion and the standard edge boundary conditions. The plate equations in question are the Kirchhoff theory [33], the Mindlin theory [4], and the Shimpi-Patel theory [12]. These theories assume that the applied surface stresses at $z= \pm h$ only have a normal component. Naturally, the orthotropic Kirchhoff and Mindlin theories simplify to the corresponding well known plate theories in the special case of an isotropic material. Consider first the Kirchhoff equation

$$
\begin{aligned}
& h \rho \partial_{t}^{2} w_{0}+\frac{h^{3}}{3}\left[-\left(\beta_{133} c_{13}-c_{11}\right) \partial_{x}^{4} w_{0}-\left(\beta_{233} c_{23}-c_{22}\right) \partial_{y}^{4} w_{0}\right. \\
& \left.-2\left[\beta_{133} c_{23}-\left(c_{126}+c_{66}\right)\right] \partial_{x}^{2} \partial_{y}^{2} w_{0}\right]=\frac{1}{2}\left(T_{z z}^{+}-T_{z z}^{-}\right) .
\end{aligned}
$$

This equation has some resemblance to the corresponding terms in Eq. (6.10), which becomes even clearer if adopting the Kirchhoff hypothesis that $\partial_{x} w_{0}=-u_{1}$ and $\partial_{y} w_{0}=-v_{1}$ in Eq. (6.10). A more detailed comparison to the present asymptotic theory requires that the three equations (6.8)-(6.10) are combined to one single equation in terms of $w_{0}$. However, due to the coupling between $\mathrm{P} / \mathrm{SV}$ and $\mathrm{SH}$ waves as discussed in Section 6.1, this procedure must be pursued carefully and is not presented here. 
Next, consider the Mindlin equations

$$
\begin{aligned}
& \kappa^{2} c_{55}\left(u_{1}+\partial_{x} w_{0}\right)+\frac{h^{2}}{3}\left[\rho \partial_{t}^{2} u_{1}+\left(\beta_{133} c_{13}-c_{11}\right) \partial_{x}^{2} u_{1}-c_{66} \partial_{y}^{2} u_{1}\right. \\
& \left.\quad+\left(\beta_{133} c_{23}-c_{126}\right) \partial_{x} \partial_{y} v_{1}\right]=0 \\
& \kappa^{2} c_{44}\left(v_{1}+\partial_{y} w_{0}\right)+\frac{h^{2}}{3}\left[\rho \partial_{t}^{2} v_{1}+\left(\beta_{233} c_{23}-c_{22}\right) \partial_{y}^{2} v_{1}-c_{66} \partial_{x}^{2} v_{1}\right. \\
& \left.\quad+\left(\beta_{233} c_{13}-c_{126}\right) \partial_{x} \partial_{y} u_{1}\right]=0 \\
& h\left[\rho \partial_{t}^{2} w_{0}-\kappa^{2} c_{55}\left(\partial_{x} u_{1}+\partial_{x}^{2} w_{0}\right)-\kappa^{2} c_{44}\left(\partial_{y} v_{1}+\partial_{y}^{2} w_{0}\right)\right]=\frac{1}{2}\left(T_{z z}^{+}-T_{z z}^{-}\right) .
\end{aligned}
$$

These equations clearly resemble the Eqs. (6.8)-(6.10), albeit there are several differences worth discussing. Firstly, the Mindlin equations omit some terms such as the last three $w_{0}$ terms in each of Eqs. (6.8)-(6.9), as well as the whole $h^{3}$ expression in Eq. (6.10). Hence, the Mindlin equations are similar to the asymptotic equations of order $N=2$. Secondly, Eq. (7.2) involves the correction factor $\kappa$ that usually is chosen so that the thickness-shear cut-off frequency coincides with the result from exact theory, that is $\kappa^{2}=\pi^{2} / 12$. Clearly, the choice of $\kappa^{2}=2 / 3$ in the first two equations and $\kappa^{2}=1$ in the last equation would result in similar coefficients of the common terms in Eq. (7.2) and Eqs. (6.8)-(6.10). It could be noted that the Kirchhoff equation can be obtained from the Mindlin equations by omitting rotatory inertia and eliminating shear deformation terms. Moreover, in the long wave and low frequency limit, both the present asymptotic and the Mindlin theory result in the Kirchhoff equation.

The Shimpi-Patel theory is a Timoshenko-like theory that involves two independent displacement fields in the vertical direction. The displacement components in the plate plane are expressed through these two vertical fields, and involve cubic terms in the $z$ coordinate. This results in a set of two plate equations, that may be combined and rewritten in terms of the variables used in the present paper. Hence, the Shimpi-Patel plate equations become

$$
\begin{aligned}
& c_{55}\left(\partial_{x} u_{1}+\partial_{x}^{2} w_{0}\right)+c_{44}\left(\partial_{y} v_{1}+\partial_{y}^{2} w_{0}\right) \\
& +\frac{h^{2}}{42}\left[17 \rho \partial_{t}^{2} \partial_{x} u_{1}+17 \rho \partial_{t}^{2} \partial_{y} v_{1}+17\left(\beta_{133} c_{13}-c_{11}\right) \partial_{x}^{3} u_{1}+17\left(\beta_{233} c_{23}-c_{22}\right) \partial_{y}^{3} v_{1}\right. \\
& +\left(17\left(\beta_{233} c_{13}-c_{12}\right)-34 c_{66}\right) \partial_{x} \partial_{y}^{2} u_{1}+\left(17\left(\beta_{133} c_{23}-c_{12}\right)-34 c_{66}\right) \partial_{x}^{2} \partial_{y} v_{1} \\
& -4 \rho \partial_{t}^{2} \partial_{x}^{2} w_{0}-4 \rho \partial_{t}^{2} \partial_{y}^{2} w_{0}-4\left(\beta_{133} c_{13}-c_{11}\right) \partial_{x}^{4} w_{0}-4\left(\beta_{233} c_{23}-c_{22}\right) \partial_{y}^{4} w_{0} \\
& \left.+\left(16 c_{66}+8 c_{12}-8 \beta_{133} c_{23}\right) \partial_{x}^{2} \partial_{y}^{2} w_{0}\right]=0 \\
& h\left[\rho \partial_{t}^{2} w_{0}-2 / 3 c_{55}\left(\partial_{x} u_{1}+\partial_{x}^{2} w_{0}\right)-2 / 3 c_{44}\left(\partial_{y} v_{1}+\partial_{y}^{2} w_{0}\right)\right] \\
& +\frac{h^{3}}{315}\left[-\left(\beta_{133} c_{13}-c_{11}\right) \partial_{x}^{4} w_{0}-\left(\beta_{233} c_{23}-c_{22}\right) \partial_{y}^{4} w_{0}-\rho \partial_{t}^{2} \partial_{x}^{2} w_{0}-\rho \partial_{t}^{2} \partial_{y}^{2} w_{0}\right. \\
& +\left(4 c_{66}+2 c_{12}-2 \beta_{133} c_{23}\right) \partial_{x}^{2} \partial_{y}^{2} w_{0}-\rho \partial_{t}^{2} \partial_{x} u_{1}-\rho \partial_{t}^{2} \partial_{y} v_{1} \\
& -\left(\beta_{133} c_{13}-c_{11}\right) \partial_{x}^{3} u_{1}-\left(\beta_{233} c_{23}-c_{22}\right) \partial_{y}^{3} v_{1} \\
& \left.+\left(2 c_{66}+c_{12}-\beta_{133} c_{23}\right) \partial_{x} \partial_{y}^{2} u_{1}+\left(2 c_{66}+c_{12}-\beta_{133} c_{23}\right) \partial_{x}^{2} \partial_{y} v_{1}\right]=\frac{1}{2}\left(T_{z z}^{+}-T_{z z}^{-}\right) .
\end{aligned}
$$


Here, the fields in the plate plane are related through $\partial_{y} u_{1}=\partial_{x} v_{1}$. Equation (7.3) corresponds to the sum of Eq. (6.8) differentiated with respect to $x$ and Eq. (6.9) differentiated with respect to $y$. Hereby, these two equations involve the same sort of derivative orders, although the coefficients in the $h^{2}$ term differ. Equation (7.4) corresponds to Eq. (6.10), but there are several differences in the coefficients as well as the omission of the $\partial_{t}^{4} w_{0}$ term in Eq. (7.4).

Concerning the standard edge conditions, the Kirchhoff equation states that two conditions should be prescribed at each edge. At the edges $x= \pm a$

$$
\begin{array}{lll}
w_{0}=0 & \text { or } & c_{11} \partial_{x}^{3} w_{0}+\left(c_{12}+4 c_{66}\right) \partial_{x} \partial_{y}^{2} w_{0}=0 \\
\partial_{x} w_{0}=0 & \text { or } & c_{11} \partial_{x}^{2} w_{0}+c_{12} \partial_{y}^{2} w_{0}=0,
\end{array}
$$

and at the edges $y= \pm b$

$$
\begin{array}{lll}
w_{0}=0 & \text { or } & c_{22} \partial_{y}^{3} w_{0}+\left(c_{12}+4 c_{66}\right) \partial_{x}^{2} \partial_{y} w_{0}=0, \\
\partial_{y} w_{0}=0 & \text { or } \quad c_{22} \partial_{y}^{2} w_{0}+c_{12} \partial_{x}^{2} w_{0}=0 .
\end{array}
$$

Naturally, these may be interpreted as homogeneous boundary conditions for the displacement, bending moment, angle and effective shear force, respectively. For the Mindlin equations, the three boundary conditions at each edge $x= \pm a$ read

$$
\begin{array}{lll}
u_{1}=0 & \text { or } & \left(c_{11}-\beta_{133} c_{13}\right) \partial_{x} u_{1}+\left(c_{12}-\beta_{133} c_{23}\right) \partial_{y} v_{1}=0 \\
v_{1}=0 & \text { or } & c_{66}\left(\partial_{y} u_{1}+\partial_{x} v_{1}\right)=0 \\
w_{0}=0 & \text { or } & c_{55}\left(u_{1}+\partial_{x} w_{0}\right)=0 .
\end{array}
$$

and at the edges $y= \pm b$

$$
\begin{array}{lll}
v_{1}=0 & \text { or } & \left(c_{22}-\beta_{233} c_{23}\right) \partial_{y} v_{1}+\left(c_{12}-\beta_{233} c_{13}\right) \partial_{x} u_{1}=0, \\
u_{1}=0 & \text { or } \quad c_{66}\left(\partial_{y} u_{1}+\partial_{x} v_{1}\right)=0, \\
w_{0}=0 & \text { or } \quad c_{44}\left(v_{1}+\partial_{y} w_{0}\right)=0 .
\end{array}
$$

These conditions are seen to be very similar to the asymptotic ones given in Eqs. (6.15)(6.20). In the case of the Shimpi-Patel theory, the four boundary conditions at each edge presented in [12] can not directly be rewritten in terms of the displacement terms used in the present paper. This could possibly be resolved by rearranging terms when adopting the Hamilton's principle [12], but this is not pursued here.

\section{NumericAl RESUlts}

The orthotropic plate equations are validated for the antisymmetric motion by investigating dispersion relations for time harmonic waves in an infinite plate, together with displacement and stress distribution plots. Moreover, eigenfrequencies are calculated for a finite square plate that is simply supported. The comparisons are in all cases made with exact threedimensional theory and the approximate theories discussed above due to Kirchhoff, Mindlin and Shimpi-Patel. The studied plate material is a graphite-epoxy (AS/3501) studied in [3], where $c_{11}=141.1 \mathrm{GPa}, c_{12}=c_{13}=5.4 \mathrm{GPa}, c_{22}=c_{33}=12.0 \mathrm{GPa}, c_{23}=6.0 \mathrm{GPa}$,

$c_{44}=6.2 \mathrm{GPa}, c_{55}=c_{66}=7.1 \mathrm{GPa}$. Introduce the dimensionless frequency $\Omega=\omega h \sqrt{\rho / c_{33}}$, adopting the time factor $\mathrm{e}^{-\mathrm{i} \omega t}$. 


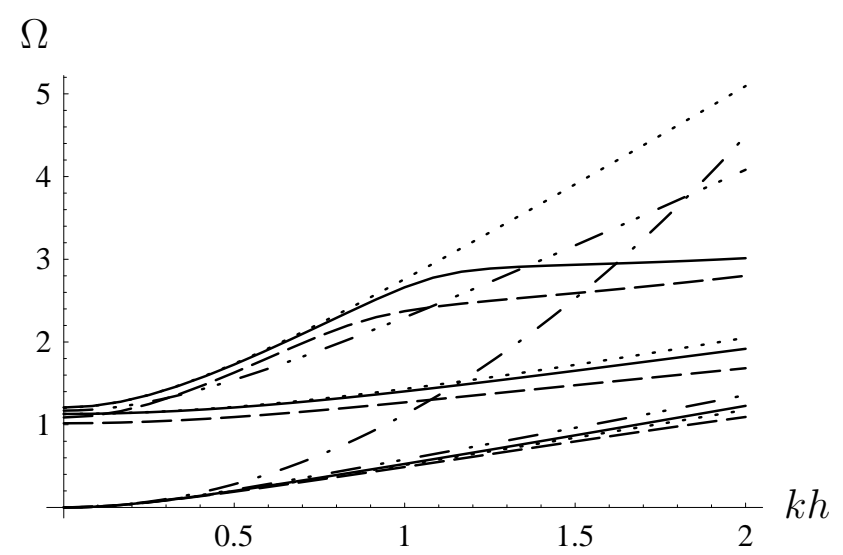

FiguRE 2. Dispersion curves:

— Exact, $---h^{3}, \cdots$ Mindlin, - - - Kirchhoff, - - - Shimpi-Patel.

Consider free waves propagating in a direction $45^{\circ}$ to the $x$ axis, where the wave number in the direction of propagation is denoted by $k$. This case is chosen as the $\mathrm{P} / \mathrm{SV}$ and the SH modes hereby couple, contrary to the situation for waves propagating along the crystal axes. Figure 2 presents dispersion curves for the asymptotic plate equations of order $N=3$ from Eqs. (6.8)-(6.10) (in the figure texts called $h^{3}$ ) together with the solutions due to exact, Kirchhoff, Mindlin and Shimpi-Patel theories. There are three sets of curves in the figure where the first one corresponds to the lowest bending mode. All approximate theories model this mode quite well for low wave numbers, albeit the Kirchhoff theory clearly deviates for higher wave numbers. The second mode may be denoted a pseudo $\mathrm{SH}$ mode even though there is coupling between the $\mathrm{P} / \mathrm{SV}$ and the $\mathrm{SH}$ modes in this direction of propagation. This mode is not modeled by the Kirchhoff or the Shimpi-Patel theory. It could be seen that the two lowest modes are best captured by the Mindlin theory. However, this does not necessarily mean that the displacements and stresses are accurately predicted, which will be seen below. For the second mode, the accurate behavior using the Mindlin theory is mainly due to the choice of the correction factor, which here results in the correct cut-off frequency. The third mode displayed corresponds to the second bending mode. Here it is seen that the asymptotic $h^{3}$ theory correctly follows the change in slope contrary to the other approximate theories. It is noted that by using higher order asymptotic plate equations such as $N=7$, the resulting plots are indistinguishable from the exact curves in the presented domain.

Next consider displacement and stress distribution plots for the specific frequency $\Omega=$ 0.5. According to the dispersion curves in Fig. 2 there exists only one real solution ( $k h \approx 0.96$ according to exact theory), corresponding to the first bending mode. Here, the displacements and stresses are either real or imaginary. The results are plotted as functions of the dimensionless thickness coordinate $z / h$, where only the upper half $z \geq 0$ is shown due to symmetry. The fields are normalized so that the vertical displacement in the middle of the plate equals unity, $w(z=0)=1$. Figures $3-4$ show the three displacement components, where the present asymptotic $h^{3}$ theory renders results that are generally equally or more accurate than the other approximate theories. Note the cubic behavior for the present and the Shimpi-Patel theories in Fig. 3, compared to the linear variation using Kirchhoff and Mindlin. Due to the definitions used for obtaining the horizontal displacements in the 


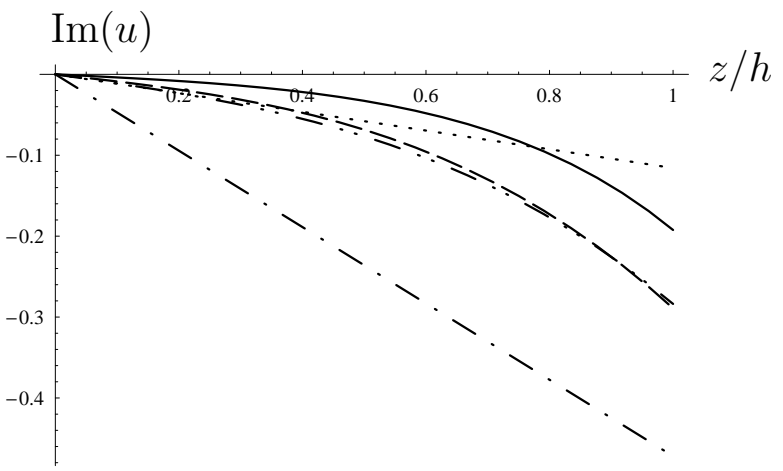

(a) Horizontal displacement in the $x$ direction.

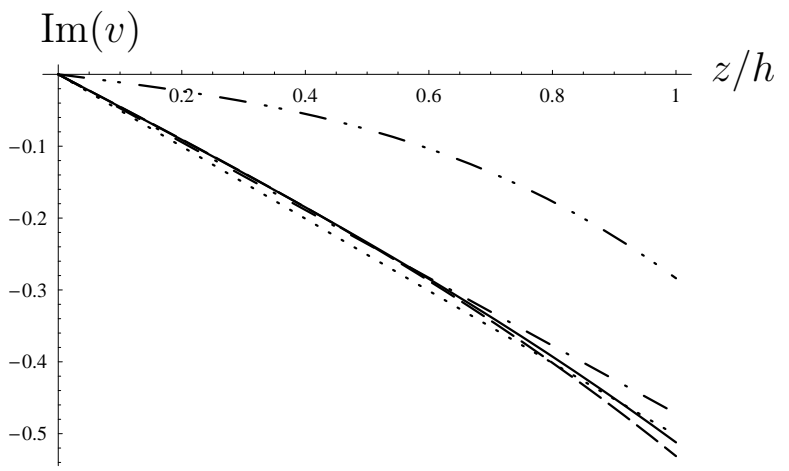

(b) Horizontal displacement in the $y$ direction.

FiguRE 3. Horizontal displacements for the first mode when $\Omega=0.5$ :

— Exact, --- $h^{3}, \cdots$ Mindlin, - - - Kirchhoff, - . - Shimpi-Patel.

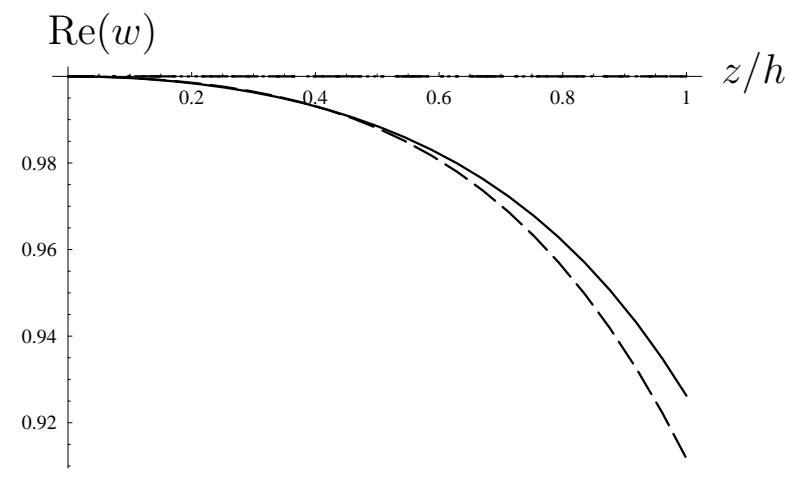

Figure 4. Vertical displacement for the first mode when $\Omega=0.5$ :

— Exact, $---h^{3}, \cdots$ Mindlin, - - - Kirchhoff, $-\cdots$ - Shimpi-Patel.

Kirchhoff and the Shimpi-Patel theories, the two curves in Figs. 3(a)-3(b) are identical for each theory. All three approximate theories used for comparison assume constant vertical displacements, lying on top of the horizontal axis in Fig. 4.

The stress distributions are presented in Figs. 5-7. Note that for the Kirchhoff, Mindlin and Shimpi-Patel theories, an indirect method is used to calculate the stresses $T_{x z}, T_{y z}, T_{z z}$ $[3,12]$. This is obtained by first calculating $T_{x x}, T_{y y}, T_{x y}$ using the constitutive relations Eq. (2.3) (the direct method). These results are then substituted into the equations of motion Eq. (2.1), where the stresses $T_{x z}, T_{y z}, T_{z z}$ are obtained adopting integration over the plate thickness (the indirect method). For $T_{x z}, T_{y z}$ the constants of integration are chosen so as to fulfil shear stress free surfaces. For $T_{z z}$ the constant of integration for each theory can not be chosen so as to give normal stress free surfaces on both sides. Instead, the constant is chosen so as to give zero normal stress in the middle of the plate, resulting in an antisymmetric stress distribution. The described indirect method hereby results in significantly improved stress distribution curves for $T_{x z}, T_{y z}, T_{z z}$ when compared to the direct method. This is especially the case for the Kirchhoff and Mindlin theories, where the direct method would result in constant shear stresses $T_{x z}, T_{y z}$ and linear normal stresses $T_{z z}$ over the plate thickness. The indirect method is here not applied to the asymptotic $h^{3}$ theory, as the resulting additional higher order terms would not be in full accordance with the adopted systematic truncation process. 


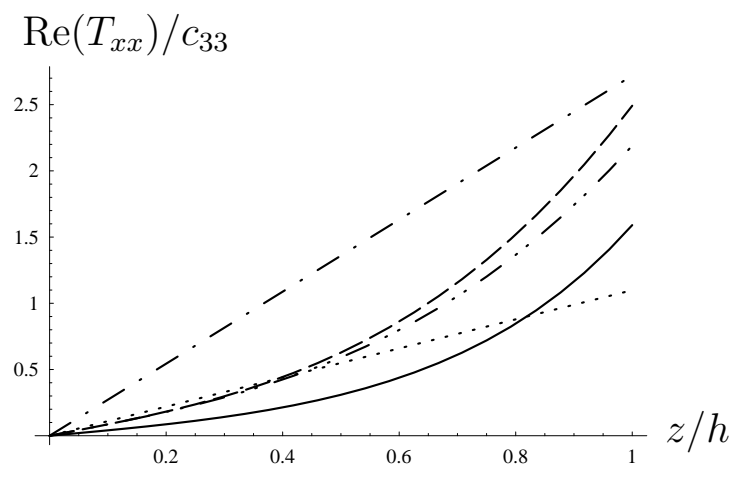

(a) Normal stress in the $x$ direction.

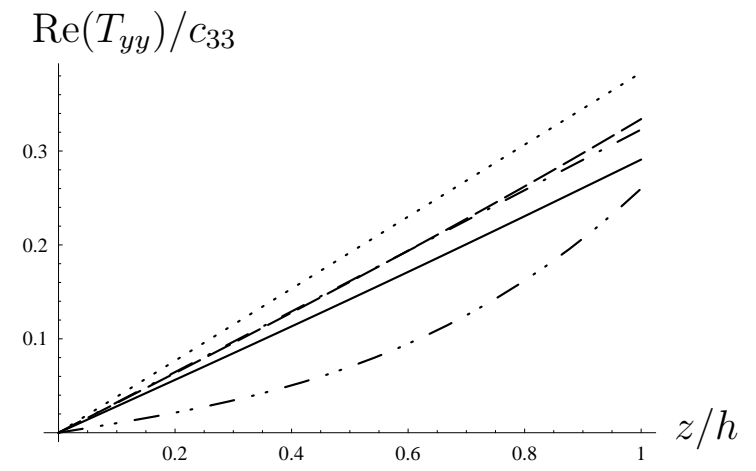

(b) Normal stress in the $y$ direction.

Figure 5. Stresses for the first mode when $\Omega=0.5$ :

— Exact, --- $h^{3}, \ldots$ Mindlin, - - Kirchhoff, - . - Shimpi-Patel.

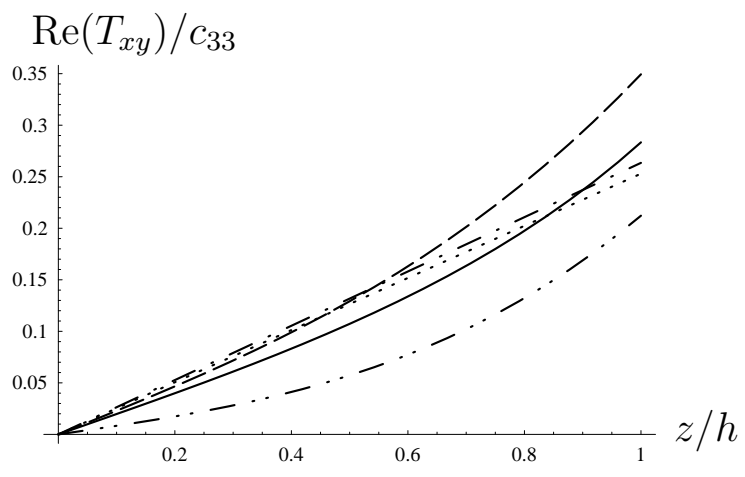

(a) Shear stress $T_{x y}$.

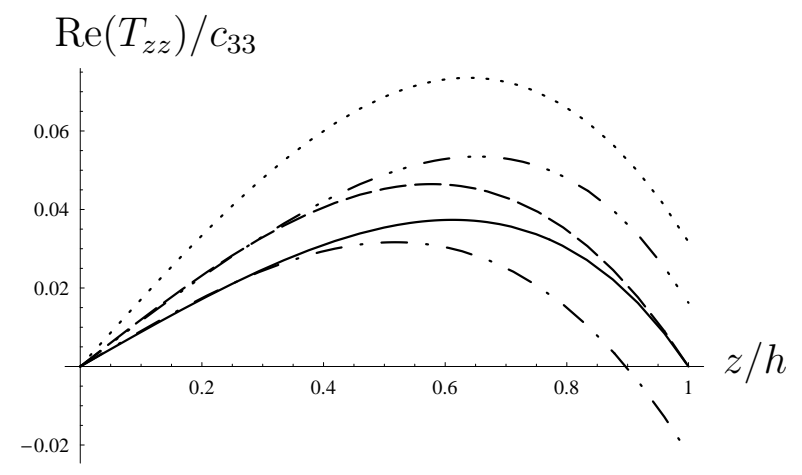

(b) Normal stress in the $z$ direction.

FiguRE 6. Stresses for the first mode when $\Omega=0.5$ :

— Exact, --- $h^{3}, \cdots$ Mindlin, $-\cdots-$ Kirchhoff, $-\cdots-$ Shimpi-Patel.

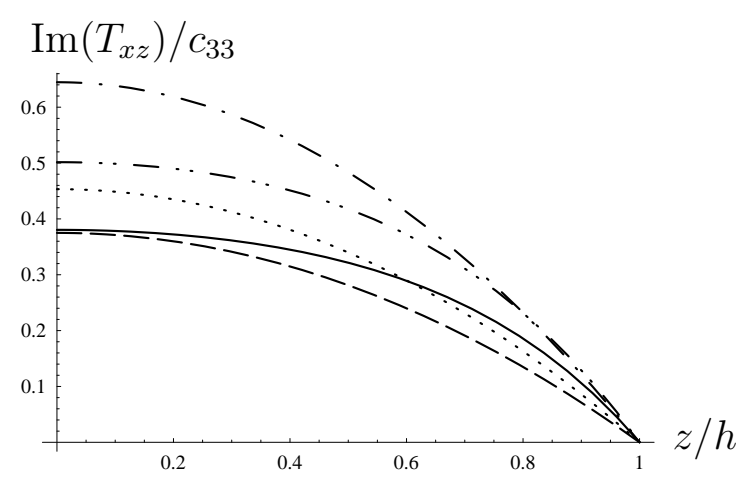

(a) Shear stress $T_{x z}$.

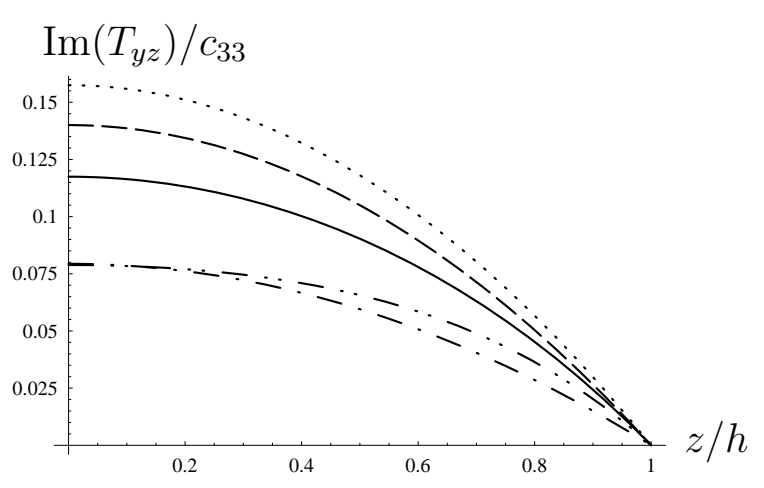

(b) Shear stress $T_{y z}$.

Figure 7. Stresses for the first mode when $\Omega=0.5$ :

— Exact, --- $h^{3}, \cdots$ Mindlin, $-\cdots-$ Kirchhoff, $-\cdots-$ Shimpi-Patel.

Note that if the indirect method is adopted using the asymptotic theory and such additional higher order terms are ignored, the same expressions are obtained as for the direct method. The corresponding situation does not occur for the other approximate theories, 
where the truncated stresses using the indirect method is different from the stresses using the direct method. The figures for $T_{x x}, T_{y y}, T_{x y}$ show that no specific theory is the most accurate for all these cases, albeit the overall behavior is best captured by the asymptotic $h^{3}$ theory. Considering $T_{x z}, T_{y z}, T_{z z}$, these stresses are in all cases best modeled through the asymptotic $h^{3}$ theory. Note that the normal stress boundary condition is only fulfilled by the asymptotic theory. As have been mentioned before, the surface boundary conditions are always automatically fulfilled using the asymptotic theory.

Consider next the displacement and stress distribution plots for the second and third modes. The chosen frequencies are $\Omega=1.5$, which for exact theory corresponds to $k h \approx 1.20$ for the second mode, and $\Omega=2$, which for exact theory corresponds to $k h \approx 0.65$ for the third mode. Plots are presented for the dominating horizontal displacement, the dominating shear stress, the vertical displacement and the normal stress. The fields are normalized such that the corresponding dominating horizontal displacement equals unity at the top surface. For the second mode, presented in Figs. 8-9, it is clear that the asymptotic $h^{3}$ theory is superior to the Mindlin theory.

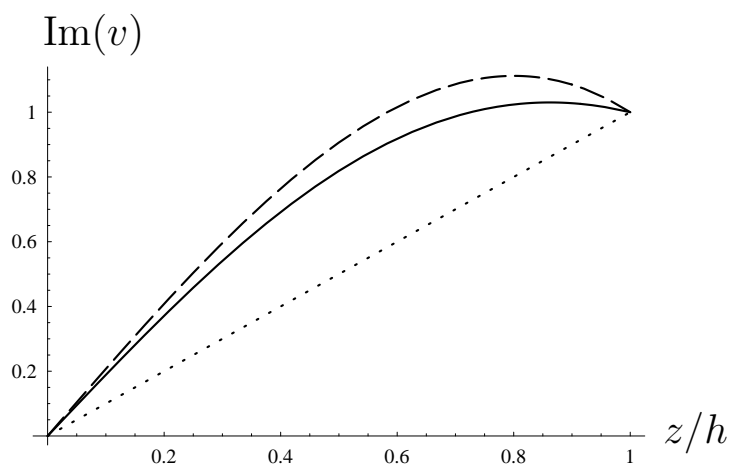

(a) Horizontal displacement in the $y$ direction.

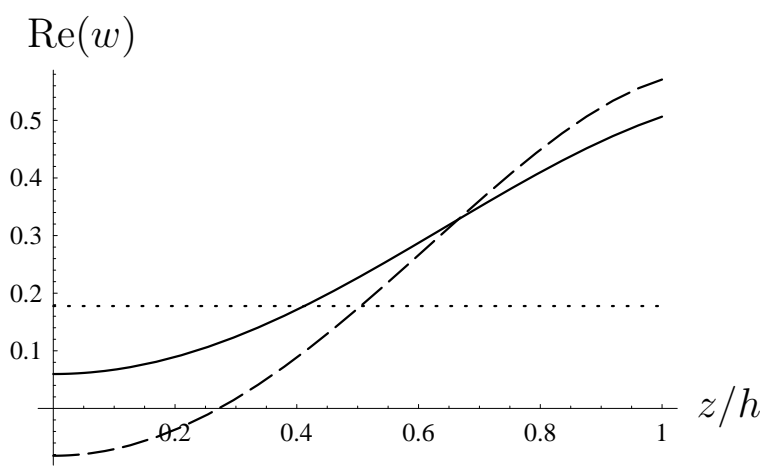

(b) Vertical displacement.

Figure 8. Displacements for the second mode when $\Omega=1.5$ :

Exact, $---h^{3}, \cdots$ Mindlin.

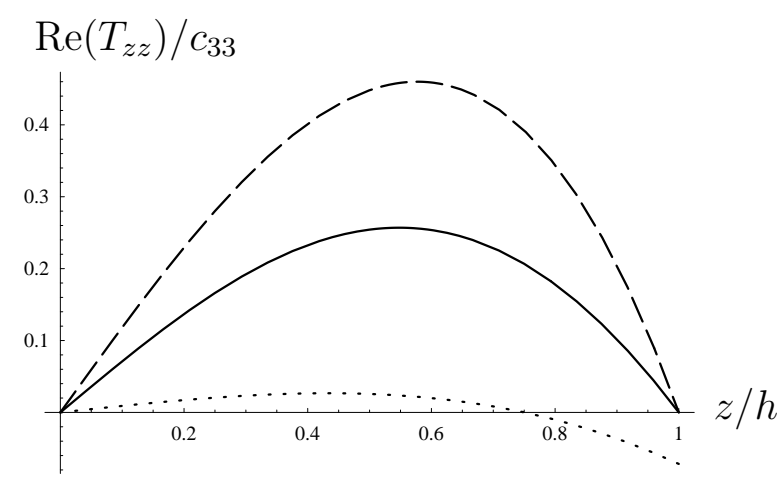

(a) Normal stress in the $z$ direction.

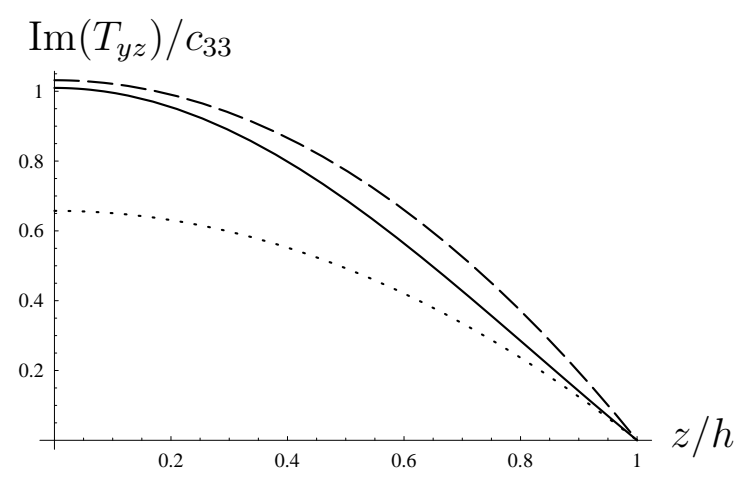

(b) Shear stress $T_{y z}$.

FiguRE 9. Stresses for the second mode when $\Omega=1.5$ :

Exact, $---h^{3}, \ldots$ Mindlin. 


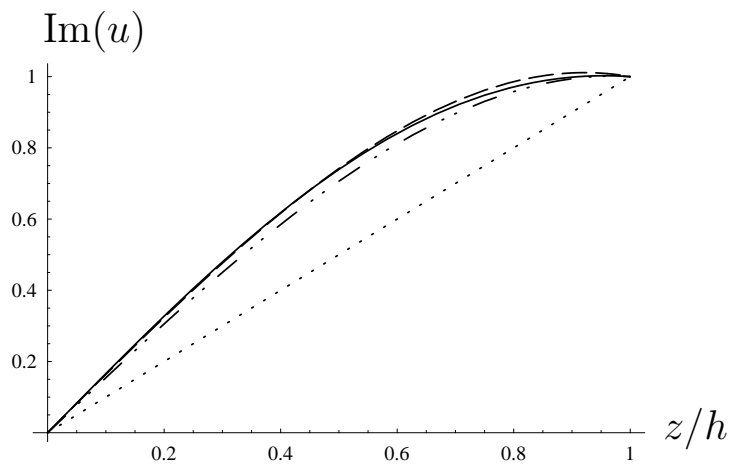

(a) Horizontal displacement in the $x$ direction.

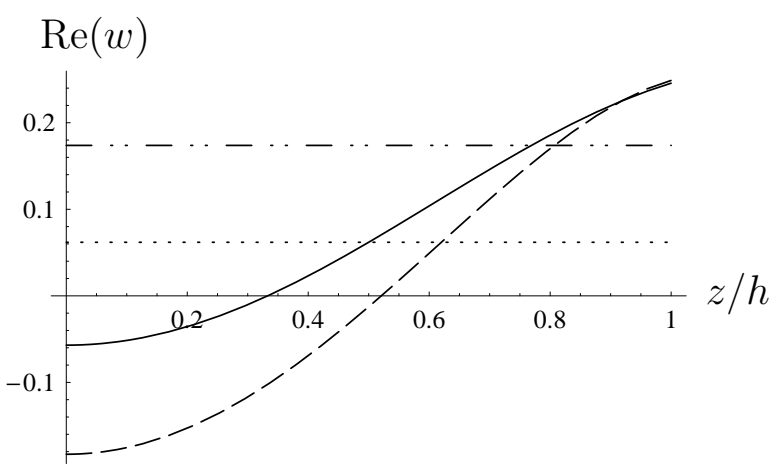

(b) Vertical displacement.

FiguRE 10. Displacements for the third mode when $\Omega=2$ :

- Exact, $---h^{3}, \cdots$ Mindlin, - . - Shimpi-Patel.

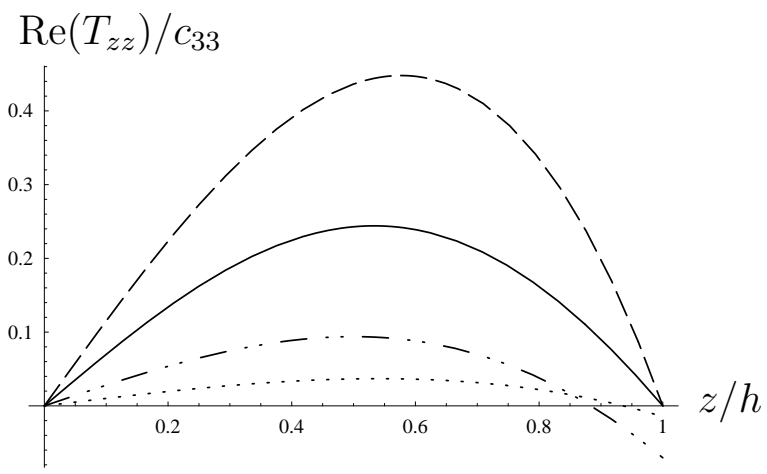

(a) Normal stress in the $z$ direction.

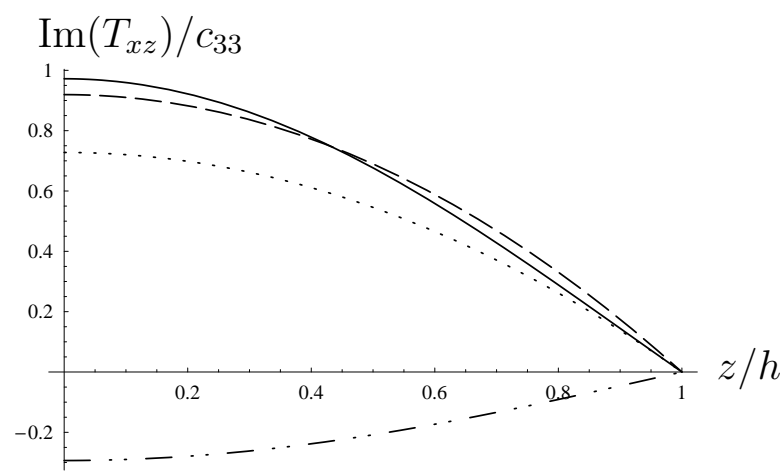

(b) Shear stress $T_{x z}$.

Figure 11. Stresses for the second mode when $\Omega=2$ :

- Exact, $---h^{3}, \cdots$ Mindlin, - - - Shimpi-Patel.

A similar behavior appears for the third mode in Figs. 10-11, which also includes the Shimpi-Patel theory. This latter theory behaves very well for the $u$ displacement in Fig. 10(a). However, it is worth mentioning that the result for the $v$ displacement (not shown here) is much less favorable for the Shimpi-Patel theory. In fact, the exact result gives that the magnitude of the $v$ displacement is less than one tenth of the $u$ displacement, while the Shimpi-Patel theory assumes identical displacements in both horizontal directions. The peculiar behavior of the shear force $T_{x z}$ in Fig. 11(b) for the Shimpi-Patel theory stems from using the indirect method. In this particular case, the direct method renders more accurate results. As expected, the overall behavior using the approximate theories for these two higher modes in Figs. 8-11 is less accurate than for the lowest mode in Figs. 3-7.

As a final illustration of the different approximate theories, the eigenfrequencies for a quadratic simply supported plate are calculated. Table 1 presents the three lowest frequencies $\Omega_{m, n}$ in the case of $h / a=0.1$ where $h$ is half the plate thickness and $a$ is half the plate width. Here $m$ and $n$ refer to the mode numbers in the $x$ and $y$ directions, respectively. It is clear that the asymptotic $h^{3}$ theory is superior to the Kirchhoff and Shimpi-Patel theories, but less accurate than the Mindlin theory. The table also illustrates how the accuracy is improved for higher order asymptotic theories 


\begin{tabular}{|c|c||c|c|c|c|c|c|c|}
\hline \hline$m$ & $n$ & Exact & Kirchhoff & Mindlin & S-P & $h^{3}$ & $h^{5}$ & $h^{7}$ \\
\hline \hline 1 & 1 & 0.0506 & 0.0554 & 0.0504 & 0.0517 & 0.0497 & 0.0505 & 0.0506 \\
\hline 1 & 2 & 0.0765 & 0.0841 & 0.0761 & 0.0779 & 0.0755 & 0.0765 & 0.0765 \\
\hline 2 & 1 & 0.1497 & 0.1999 & 0.1487 & 0.1536 & 0.1425 & 0.1493 & 0.1497 \\
\hline \hline
\end{tabular}

TABLE 1. Simply supported plate: The dimensionless eigenfrequencies $\Omega_{m, n}$ for exact theory and the approximate theories: Kirchhoff, Mindlin, ShimpiPatel (S-P), $h^{3}, h^{5}$ and $h^{7}$.

\section{Concluding REMARKS}

This paper considers plate equations for a fully anisotropic plate using a systematic power series expansion approach. In the general case, the plate equations with pertinent edge conditions become very complicated. Consequently these results are given implicitly on a compact matrix form using recursively defined differential operators. More tractable equations are given for an orthotropic material, where explicit plate equations are presented for both antisymmetric and symmetric motions when $h^{3}$ terms are included.

Analytical comparisons are made with the two classical theories of Kirchhoff and Mindlin type. A more recent plate equation developed by Shimpi-Patel is also included. The numerical results display dispersion curves, displacement and stress distributions over the plate thickness as well as eigenfrequencies for a simply supported plate. These comparisons involve the asymptotic plate equation of order $h^{3}$, the exact theory and the theories mentioned above. The results from the dispersion curves and the eigenfrequencies show that the Kirchhoff theory is clearly inferior to the other more refined theories as expected. Here the results due to the Mindlin theory are especially accurate. For the displacement and stress distributions, the asymptotic plate theory best captures the main behavior, including the exact surface stress boundary conditions.

The present plate theory may be used in a finite element environment. Such a refined theory yields more accurate results than simpler traditional equations and at the same time the number of elements in a finite element code can be heavily reduced compared to using three-dimensional elements. There are also prospects to extend the present work to other geometries, e.g. anisotropic shells. Another related topic is to study fully anisotropic piezoelectric plates; a work which is in progress.

\section{ACKNOWLEDGEMENT}

The present work is sponsored by the the Swedish Research Council and this is gratefully acknowledged.

\section{REFERENCES}

[1] H. Ekstein. High frequency vibrations of thin crystal plates. Phys. Rev., 68:11-23, 1945. 
[2] E.G. Newman and R.D. Mindlin. Vibrations of a monoclinic crystal plate. Phys. Rev., 29:1206-1218, 1957.

[3] J.N. Reddy. Mechanics of laminated composite plates: theory and analysis. CRC Press, Boca Raton, 1997.

[4] R.D. Mindlin. Thickness-shear and flexural vibrations of crystal plates. J. Appl. Phys., 22:316-323, 1951.

[5] S.J. Medwadowski. A refined theory of elastic, orthotropic plates. J. Appl. Mech., 25:437-443, 1958.

[6] P.C Yang, C.H. Norris, and Y. Stavsky. Elastic wave propagation in heterogeneous plates. Int. J. Solids Struct., 2:665-684, 1966.

[7] K.H. Lo, R.M. Christensen, and E.M. Wu. A high-order theory of plate deformation. Part 2: Laminated plates. J. Appl. Mech., 44:669-676, 1977.

[8] K.T. Sundara Raja Iyengar and S.K. Pandya. Analysis of orthotropic rectangular thick plates. Fibre Sci. Techn., 18:19-36, 1983.

[9] J.N. Reddy. A refined nonlinear theory of plates with transverse shear deformation. Int. J. Solids Struct., 20:881-896, 1984.

[10] K.P. Soldatos. On certain refined theories for plate bending. J. Appl. Mech., 55:994-995, 1988.

[11] T. Kant and G.G. Gadgil. Analysis of orthotropic plates based on three theories by segmentation method. Mech. Adv. Mat. Struct., 9:189-239, 2002.

[12] R.P. Shimpi and H.G. Patel. A two variable refined plate theory for orthotropic plate analysis. Int. J. Solids Struct., 43:6783-6799, 2006.

[13] J.D. Achenbach. An asymptotic method to analyze the vibrations of an elastic layer. J. Appl. Mech., 22:65-72, 1969.

[14] O.E. Widera. An asymptotic theory for the motion of elastic plates. Acta Mech., 9:54-66, 1970.

[15] F.I. Niordson. An asymptotic theory for vibrating plates. Int. J. Solids Struct., 15:167-181, 1979.

[16] H. Matsunaga. The application of a two-dimensional higher-order theory for the analysis of a thick elastic plate. Comp. Str., 45(4):633-648, 1992.

[17] I.T. Selezov. Hyperbolic models of wave propagation in rods, plates, and shells. Izv. RAN. Mekhanika Tverdogo Tela, 29:64-77, 1994.

[18] K.P. Soldatos. Generalization of variationally consistent plate theories on the basis of a vectorial formulation. J. Sound Vib., 183:819-839, 1995.

[19] N.A. Losin. Asymptotics of flexural waves in isotropic elastic plates. J. Appl. Mech., 64:336-342, 1997.

[20] A. Boström, G. Johansson, and P. Olsson. On the rational derivation of a hierarchy of dynamic equations for a homogeneous, isotropic, elastic plate. Int. J. Solids Struct., 38:2487-2501, 2001.

[21] R.D. Mindlin. High frequency vibrations of crystal plates. Q. Appl. Math., 19:51-61, 1961.

[22] Y.Y. Yu. On linear equations of anisotropic elastic plates. Quart. Appl. Math., 22:357-360, 1965.

[23] G. Johansson and A.J. Niklasson. Approximate dynamic boundary conditions for a thin piezoelectric layer. Int. J. Solids Struct., 40:3477-3492, 2003.

[24] K. Mauritsson, A. Boström, and P.D. Folkow. Modelling of thin piezoelectric layers on plates. Wave Motion, 45:616-628, 2008.

[25] P.D. Folkow and M. Johansson. Dynamic equations for fluid-loaded porous plates using approximate boundary conditions. J. Acoust. Soc. Am., 125:2954-2966, 2009.

[26] A. Boström. On wave equations for elastic rods. Z. Angew. Math. Mech., 80:245-251, 2000.

[27] P.D. Folkow and K. Mauritsson. Dynamic higher order equations for finite rods. Accepted for publication in Q. Jl Mech. Appl. Math., 2010.

[28] P.A. Martin. Waves in wood: axisymmetric waves in slender solids of revolution. Wave Motion, 40:387-398, 2004.

[29] P.A. Martin. On flexural waves in cylindrically anisotropic elastic rods. Int. J. Solids Struct., 42:2161$2179,2005$.

[30] A.M. Hägglund and P.D. Folkow. Dynamic cylindrical shell equations by power series expansions. Int. J. Solids Struct., 45:4509-4522, 2008.

[31] B.A. Auld. Acoustic Fields and Waves in Solids. Krieger, Malabar, FL, 1990.

[32] M. Johansson, P.D. Folkow, A.M. Hägglund, and P. Olsson. Approximate boundary conditions for a fluid-loaded elastic plate. J. Acoust. Soc. Am., 118:3436-3446, 2005.

[33] J.S. Rao. Dynamics of Plates. Narosa Publishing House, New Delhi, 1999. 
Department of Applied Mechanics, Chalmers University of Technology, SE-412 96 GöteBORG, SWEDEN 